\title{
A Note on An Algorithm for Interpolating Rationals
}

\author{
By Huang Yougun and Jack Williams
}

\begin{abstract}
This note considers an aspect of the simultaneous exchange algorithm for Chebyshev approximation by interpolating rationals. An example has been given by Dunham claiming to show that this algorithm may fail to produce an interpolating rational approximant. In this note we show that his example cannot occur as a step of the exchange algorithm. A second example is given where Dunham's objection to the algorithm is valid.
\end{abstract}

1. Introduction. Williams [4] and subsequently Taylor and Williams [3] developed a theory and an algorithm for best Chebyshev approximation of decay-type functions by an oscillation factor times a negative power of a generalized polynomial. The algorithm, a variant of the exchange algorithm, requires at each step the solution of a system of nonlinear levelling equations. These equations are shown to have a unique solution in [4], but a pole-free solution cannot be guaranteed [4, p. 202]. Dunham [1] obtained a result in which he constructed a function for which these levelling equations have no pole-free solution. In this note we show that Dunham's construction cannot arise as a step in the exchange algorithm; an example where it can occur is given.

2. Approximation by Interpolating Rationals. In the notation of [4] let $D[0, b]$, $0<b<\infty$, denote the continuous functions of the form $f(x)=B(x) g(x), g \in$ $C[0, b], g>0 \forall x \in[0, b]$, where $B(x)$ satisfies $B\left(x_{\nu}\right)=0$ for a finite number of $x_{\nu} \in[0, b]$. Given $f \in D[0, b]$ let $V$ denote the class of approximating functions $F(A, x)=B(x) / L(A, x)^{P}, x \in[0, b]$ which satisfy

(a) $L(A, x)=\sum_{r=1}^{n} a_{r} \phi_{r}(x), \phi_{1} \equiv 1, \phi_{r} \in C[0, b], r=2,3, \ldots, n$, and $\left\{\phi_{1}, \phi_{2}, \ldots\right.$, $\left.\phi_{n}\right\}$ forms a Chebyshev set on $[0, b]$,

(b) $L(A, x)>0 \forall x \in[0, b]$

(c) $0<p<\infty$.

Let $\|\cdot\|$ denote the Chebyshev norm on $C[0, b]$. Then the problem is to determine the best approximation $F\left(A^{*}, x\right) \in V$ so that $\left\|f-F\left(A^{*}, x\right)\right\| \leqslant\|f-F(A, x)\| \forall$ $F(A, x) \in V$. Sufficient conditions for the existence of $F\left(A^{*}, x\right)$ are given in [3] and characterization and uniqueness in [4]; also see Schmidt [2]. In addition [4] describes an exchange algorithm for computing $F\left(A^{*}, x\right)$ in which a sequence of approximations $F\left(A_{k}, x\right) \in V, k=0,1,2, \ldots$, is obtained by solving the nonlinear levelling equations

$$
\left|B\left(x_{r}^{(k)}\right)\right|\left\{g\left(x_{r}^{(k)}\right)-L\left(A_{k}, x_{r}^{(k)}\right)^{-P}\right\}=(-)^{r} \lambda_{k}, \quad r=1,2, \ldots, n+1,
$$


where $X^{(k)}:=\left\{x_{1}^{(k)}, x_{2}^{(k)}, \ldots, x_{n+1}^{(k)}\right\} \subset[0, b]$ is the current reference. Zeros of $B(x)$ cannot be contained in $X^{(k)}$. These equations have a unique solution [4] for $\left(A_{k}, \lambda_{k}\right)$ but there is the possibility that $F\left(A_{k}, x\right) \notin V$.

TheOREM (DUNhaM [1]). Let $L(C, x)$ change sign on $[0, b]$ and be positive on $a$ subinterval $I$ on which $B(x)$ is nonzero. Let $x_{1}, x_{2}, \ldots, x_{n+1}$ be distinct points in $I$. There exists $g>0$ such that $(C, 0)$ is a solution to

$$
\left|B\left(x_{i}\right)\right|\left\{g\left(x_{i}\right)-L\left(A, x_{i}\right)^{-P}\right\}=(-)^{i} \lambda, \quad i=1,2, \ldots, n+1,
$$

and no pole free solution exists.

In the proof Dunham constructs a positive $g$ such that $g\left(x_{i}\right)=L\left(C, x_{i}\right)^{-P}, i=1$, $2, \ldots, n+1$, and so $(C, 0)$ is a solution. We shall now use this construction and associate the $x_{1}, x_{2}, \ldots, x_{n+1}$ with the reference $X^{(k)}$ and $\lambda$ with $\lambda_{k}$; we note that $X^{(k)} \subset I$ on which $L(C, x)$ is positive and $B(x)$ is nonzero. Assuming $F\left(A_{k-1}, x\right)$ $\in V$, then from the exchange algorithm:

$$
g(x)-L\left(A_{k-1}, x\right)^{-P} \text { alternates in sign on } X^{(k)} .
$$

Now suppose $g$ satisfies Dunham's construction, then

$$
\begin{aligned}
g\left(x_{r}^{(k)}\right)-L\left(A_{k-1}, x_{r}^{(k)}\right)^{-P} & =L\left(C, x_{r}^{(k)}\right)^{-P}-L\left(A_{k-1}, x_{r}^{(k)}\right)^{-P} \\
& =(-1)^{r} u_{r}, \quad r=1,2, \ldots, n+1,
\end{aligned}
$$

where $u_{r} \neq 0$ and are all of the same sign. Hence $L(C, x)-L\left(A_{k-1}, x\right)$ has at least $n$ zeros in $[0, b]$, and so the Chebyshev set hypothesis implies $L(C, x) \equiv L\left(A_{k-1}, x\right)$; this contradicts the condition on $C$.

We conclude that, apart from at the start of the algorithm, Dunham's construction is inappropriate for the setting of the exchange algorithm. We can, however, give an example where for $F\left(A_{k-1}, x\right) \in V$ there results $F\left(A_{k}, x\right) \notin V$.

A function $f \in C[0,0.7]$ will be defined and approximated by the elements $F(A, x)=x /\left(a_{1}+a_{2} x\right), B(x) \equiv x, a_{1}+a_{2} x>0 \forall x \in[0,0.7]$. The function $f$ and its best approximation are illustrated in the figure. It will be clear that other constructions for $f$ are possible.

Consider $y_{1}(x)=2 x /(1+x), x \in[0,0.7]$, and let $A, B$ and $C$ have respective coordinates
A: $(1 / 4,3 / 5)=\left(1 / 4, y_{1}(1 / 4)+1 / 5\right)$,
B: $(3 / 10,17 / 65)=\left(3 / 10, y_{1}(3 / 10)-1 / 5\right)$,
C: $(7 / 10,87 / 85)=\left(7 / 10, y_{1}(7 / 10)+1 / 5\right)$.

Let $y_{2}(x)=15 x /(14-5 x), x \in[0,0.7]$, and let $D$ and $E$ have coordinates

D: $(2 / 5,2 / 5)=\left(2 / 5, y_{2}(2 / 5)-1 / 10\right)$,

$\mathrm{E}:(154 / 355,3 / 5)=\left(154 / 355, y_{2}(154 / 355)+1 / 20\right)$.

From $(0,0)$ to $A, A$ to $B$ and $D$ to $E f(x)$ are straight line segments. From $B$ to $D$ choose a continuous $f$ to satisfy

$$
y_{2}(x)-1 / 10<f(x)<y_{2}(x) \text { and } f(x)>y_{1}(x)-1 / 5, \quad x \in(3 / 10,2 / 5) \text {; }
$$

since $y_{2}(3 / 10)-1 / 10<f(3 / 10)$ such a construction is possible. From $E$ to $C$ choose a continuous $f$ to satisfy for $x \in(154 / 355,7 / 10)$,

$$
y_{2}(x)<f(x)<y_{2}(x)+1 / 20 \text { and } f(x)<y_{1}(x)+1 / 5 .
$$


Since $y_{2}(7 / 10)+1 / 20>f(7 / 10)$ such a construction is possible. For $x \in[0,0.7]$ there follows $y_{1}(x)-1 / 5 \leqslant f(x) \leqslant y_{1}(x)+1 / 5$.

From the characterization theorem [4, Theorem 3.3] it is clear that $f(x)$ has a best approximation $F\left(A^{*}, x\right)=y_{1}(x)$ with extremal set corresponding to the points $A, B$ and $C$ and error 1/5; see the figure. Suppose now $F\left(A^{(k-1)}, x\right)=y_{2}(x)(k$ may be 1 , corresponding to the initial approximation in the exchange algorithm) and consider the error $f(x)-F\left(A^{(k-1)}, x\right)$. At the points $A, B, D, E$ and $C$ this error takes the respective values $26 / 85,-14 / 195,-1 / 10,1 / 20$ and $2 / 85$. Hence, from the construction of $f(x)$, it follows that the exchange algorithm will select a new reference corresponding to the points $A, D$ and $E$. Then $F\left(A^{(k)}, x\right)=1 / 2=x / 2 x$ $\notin V$.

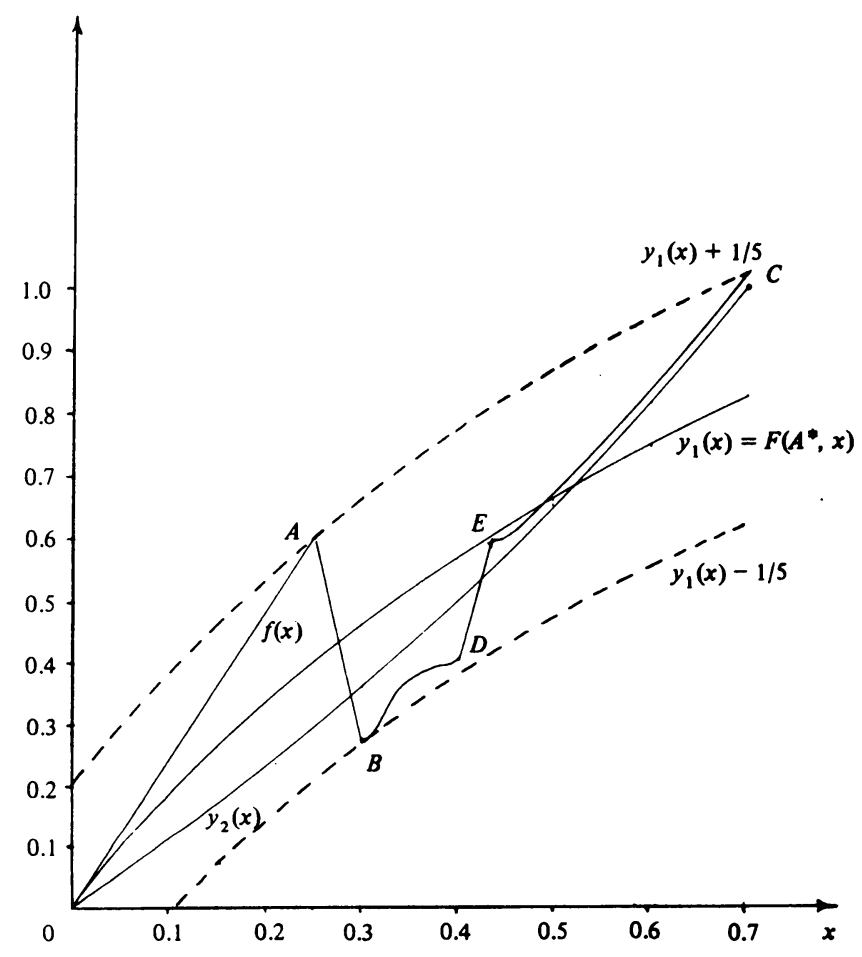

Figure 1

Computer Science Application Office

Electronic Engineering Department

Shen Yang Institute of Mechanical and Electrical Engineering

Peoples Republic of China

Department of Mathematics

University of Manchester

Manchester M13 9PL, England

1. C. B. Dunham, “A difficulty in Williams' algorithm for interpolating rationals,” Math. Comp., v. 29. 1975, pp. 552-553.

2. D. SCHмiDT, "An existence theorem for Chebyshev approximation by interpolating rationals," $J$. Approx. Theory, v. 27, 1979, pp. 146-152.

3. G. D. TAYLOR \& J. Williams, "Existence questions for the problem of Chebyshev approximation by interpolating rationals," Math. Comp., v. 28, 1974, pp. 1097-1103.

4. J. Williams, “Numerical Chebyshev approximation by interpolating rationals," Math. Comp., v. 26, 1972, pp. 199-206. 